\title{
Efficiently Mining Unordered Trees
}

\author{
Mostafa Haghir Chehreghani \\ Department of Computer Science \\ Katholieke Universiteit Leuven, Belgium \\ Mostafa.HaghirChehreghani@cs.kuleuven.be
}

\begin{abstract}
Frequent tree patterns have many applications in different domains such as XML document mining, user web $\log$ analysis, network routing and bioinformatics. In this paper, we first introduce three new tree encodings and accordingly present an efficient algorithm for finding frequent patterns from rooted unordered trees with the assumption that children of every node in database trees are identically labeled. Then, we generalize the method and propose the UITree algorithm to find frequent patterns from rooted unordered trees without any restriction. Compared to other algorithms in the literature, UItree manages occurrences of a candidate tree in database trees more efficiently. Our extensive experiments on both real and synthetic datasets show that UITree significantly outperforms the most efficient existing works on mining unordered trees.
\end{abstract}

Keywords-Frequent tree patterns, rooted unordered trees, tree encoding, candidate generation, frequency counting.

\section{INTRODUCTION}

The problem of finding frequent patterns from a database of graphs has several important applications in different areas like bioinformatics, user web log analysis, web mining and network routing. It is also a fundamental problem in many other data mining tasks such as association rule mining, classification and clustering. Although in recent years finding condensed representations of frequent patterns (for example closed patterns) has found more interest, developing efficient algorithms for finding frequent patterns is still important, because the efficiency of the algorithms of finding condensed representations depends on the efficiency of the frequent pattern mining algorithms.

Trees are an important class of graphs having many applications such as XML documents, World Wide Web and computer vision. In this paper, we focus on rooted unordered trees.

Several algorithms are in the literature for finding frequent patterns from rooted unordered trees. Asai et al. and Nijssen et al. proposed Unot [2] and uFreqt [14], respectively. For frequency counting, Unot uses an occurrence list based approach in which each occurrence is stored in $\mathrm{O}(L)$ space ( $L$ is the size of the candidate tree). uFreqt uses a different occurrence list based approach for frequency counting that its size is bounded by the product of the size of the database and the size of the candidate. The more recent well known algorithm for mining induced patterns is HybridTreeMiner [6] which uses the breadth-first canonical form. However, for frequency counting, HybridTreeMiner deals with the occurrence-list of every candidate. PathJoin [25] assumes that children of every node are labeled identically and finds maximal patterns. More recently, UNI3 [8] was proposed for unordered candidate generation, however, it counts ordered frequency of every candidate.

In [4], the authors introduced two tree encodings for rooted ordered trees and accordingly, developed an efficient algorithm for finding frequent patterns from rooted ordered trees. However, the method of [4] can not easily be extended to mine unordered trees. In this paper, we follow the idea of storing a few encodings, instead of dealing with the occurrences of a candidate tree in a database tree, and perform the frequency counting step by means of efficient comparisons over these encodings. Our key contributions are as follows:

- We introduce three novel tree encodings, and accordingly, develop a new frequency counting algorithm for mining unordered trees with unique labels on children of every node.

- Then, we generalize the method and propose the UITree algorithm to find frequent patterns from rooted unordered trees without any restriction.

- We perform extensive experiments on both synthetic and real datasets and show that UITree significantly outperforms the well-known algorithms in the literature.

The rest of this paper is organised as follows. In Section II, preliminaries and definitions related to the problem of finding frequent tree patterns are given. Section III describes the proposed algorithm including the candidate generation method, the new tree encodings, and the frequency counting method. We experimentally evaluate the efficiency of UITree and compare it against the existing efficinet methods in Section IV. In Section V, we have a brief overview on the related works. Finally, in Section VI, the paper is concluded and some possible extensions of the current work are presented.

\section{PRELIMINARIES AND PROBLEM STATEMENT}

A rooted labeled unordered tree $T=(V, E, L)$ is a connected DAG with $V$ as the set of nodes, $E=\{(u, v) \mid u, v \in$ $V\}$ as the set of edges, and $L$ as the set of labels. A distinguished node $r$ is considered as the root, and for any other node $v$, there is a unique path from $r$ to $v$. Further, 
there is a function $\lambda: V \rightarrow L$ which maps nodes to labels. A rooted labeled ordered tree has a left-to-right ordering among the siblings.

The degree of a node $v$ in a tree $T$, denoted by $\operatorname{deg}(T, v)$, is defined as the number of edges incident to $v$. The depth of a node $v$ in a tree $T$, denoted by $\operatorname{dep}(T, v)$, is the length of the path from the root to $v$. We say the node $v$ is in the position $n$ of a path $p$, if $\operatorname{dep}(T, v)$ is equal to $n$.

For a labeled tree $T$, Zaki's string representation, denoted by $S(T)$ is defined as follows: the labels of the nodes of $T$ are added to $S(T)$ in the preorder traversal of $T$, and when a backtracking from a child to its parent is occur a unique symbol (e.g. -1) is added to $S(T)$ [28].

For a rooted labeled unordered tree $T=(V, E, L)$, a rooted labeled unordered tree $T^{\prime}=\left(V^{\prime}, E^{\prime}, L^{\prime}\right)$ is an induced subtree of $T$, also called $T^{\prime}$ is isomorphic to a subtree of $T$, if and only if: (1) $V^{\prime} \subseteq V$, (2) $E^{\prime} \subseteq E$, and (3) $L^{\prime} \subseteq L$ and the labeling of $\bar{V}^{\prime}$ in $T$ is preserved in $T^{\prime}$. An occurrence $O^{\prime}$ of $T^{\prime}$ in $T$ is the set of nodes and edges of $T$ to which $T^{\prime}$ is mapped. We note $T^{\prime}$ might have zero, one, or several occurrences in $T . T^{\prime}$ is a subtree isomorphic of $T$ if and only if $T^{\prime}$ has at least one occurrence in $T$. Two different occurrences of $T^{\prime}$ in $T$ might share some nodes (and edges) in common, but they can not be the same, entirely.

Given a database $\mathcal{D}$ consisting of rooted labeled unordered trees and a subtree $S$, the per-tree support (or per-tree frequency) of $S$ is defined as the number of trees in $\mathcal{D}$ for which $S$ is an induced subtree. The occurrence-match support (or occurrence-match frequency) of $S$ is defined as the number of occurrences of $S$ in $\mathcal{D}$. More formally:

$$
\text { per-tree } \operatorname{support}(S)=\sum_{T \in \mathcal{D}} \operatorname{IsInduced}(S, T)
$$

and

$$
\text { occurrence-match } \operatorname{support}(S)=\sum_{T \in \mathcal{D}} \operatorname{OccNum}(S, T)
$$

where $\operatorname{IsInduced}(S, T)$ is 1 if $S$ is the induced subtree of the database tree $T$ otherwise 0 , and $\operatorname{OccNum}(S, T)$ returns the number of occurrences of $S$ in $T$. A tree is frequent if its per-tree support (occurrence-match support) is greater than or equal to a user-specified per-tree (occurrence-match) min_sup value.

The desired class of patterns can differ based on the kind of the application. In this paper, our concern is frequent induced unordered tree patterns. Both of per-tree frequency and occurrence-match frequency are allowed in this work, but we mainly concentrate on occurrence-match frequency. Through this paper, for simplicity we use the term of frequency (support) instead of occurrence-match frequency (occurrence-match support); unless we explicitly say that frequency (support) refers to per-tree frequency (per-tree support).

\section{Mining UNORDERED TREES}

In this section, first, we present the candidate generation method. Then, we introduce three new tree encodings: $s$ coding, is-coding and cs-coding, which are assigned to nodes of database trees. Next, we present a frequency counting method for unordered trees having unique labels on children of every node. Next, we generalize the frequency counting method for all rooted unordered trees. Finally, we present the UITree algorithm.

\section{A. Candidate generation}

For candidate generation, we use a variation of the wellknown rightmost path extension method. Let $C$ be a tree having $k$ nodes. We call it a $k$-tree. Using rightmost path extension, $C$ can be extended to generate new trees in two different ways. In the first way, called rn_extension, a new node $N$ is added to the rightmost node of $C$. In the second way, called rp_extension, a new node $N$ is added to a node in the rightmost path of $C$, except its rightmost node [4]. In both cases, $N$ will be the rightmost node of the generated $k+1$-tree. For ordered trees, this method of candidate generation is complete as well as non-redundant, however, for unordered trees, it might generate redundant trees. Duplicate candidates can be detected and ignored by means of a canonical form.

For $r p \_$_extension, we use the technique proposed in [29] to make the candidate generation method more efficient.

Definition 1: Two $k$-trees $C_{1}$ and $C_{2}$ are in the same equivalence class, denoted by $C_{1} \simeq C_{2}$, if they share the first $k-1$ nodes in common.

Definition 2: Assume that $C_{1}$ and $C_{2}$ are two $k$-trees and $C_{1} \simeq C_{2}$. Let $v_{2}$ be the rightmost node of $C_{2}$ and $v_{1}$ be the node in the position $\operatorname{dep}\left(C_{2}, v\right)-1$ of the righmost path of $C_{1}$. The join of $C_{1}$ and $C_{2}$, denoted by $C_{1} \odot C_{2}$ is a $k+1$-tree $C^{\prime}$ generated by adding $v_{2}$ to $C_{1}$ as the rightmost child of $v_{1}$.

Clearly, the join operation is defined if there is a node in the position $\operatorname{dep}\left(C_{2}, v\right)-1$ of the rightmost path of $C_{1}$, and this means that the following condition should be held: $\operatorname{dep}\left(C_{1}, n_{1}\right) \leq \operatorname{dep}\left(C_{2}, n_{2}\right)$, where $n_{1}$ and $n_{2}$ are the rightmost nodes of $C_{1}$ and $C_{2}$, respectively.

We note that the join operation defined in Definition 2 is not commutative, i.e. $C_{1} \odot C_{2}$ and $C_{2} \odot C_{1}$ might be different trees, or only one of them might be defined.

In summary, our method for extending a candidate $C$ is as the following:

- rp_extension of $C$ : for all trees $C^{\prime}$ such that $C \simeq C^{\prime}$ (including $C$ itself), $C \odot C^{\prime}$ is generated as a new candidate.

- rn_extension of $C$ : every frequent label is added to the rightmost node of $C$ as a child and generates a larger candidate.

In the rest of this paper, the rightmost path and the rightmost node of an unordered candidate tree refer respectively 
to the last path and the last node appended to the candidate in its extension chain.

\section{B. Three new encodings}

Let $T$ be a database tree and $\prec$ be a left-to-right ordering among children of nodes of $T$. The ordered form of $T$ with respect to $\prec$, denoted by $T \prec$, refers to the ordered tree resulted by applying $\prec$ to the nodes of $T$.

$s$-coding

Definition 3: Let $T$ be a database tree, $\prec$ be a left-toright ordering among children of nodes of $T$, and sid be an auxiliary integer initiated by 0 . For every node $v \in V(T)$, its $s$-coding with respect to $\prec$, denoted by $\mathcal{S C} \prec(T, v)$ is defined as the following:

1) if $v$ is the root of $T, \mathcal{S C}_{\prec}(T, v)$ is defined equal to 0 .

2) $T_{\prec}$ is traversed in preorder and when a node $v$ is met, if $\operatorname{deg}\left(T_{\prec}, v\right)>1$, sid is increased by 1 , and for any child $u$ of $v, \mathcal{S C}_{\prec}(T, u)$ is defined equal to the new value of sid.

All children of a node have the same $s$-coding. Since the nodes of the tree are traversed in preorder, when assigning $s$-coding to the children of a node, its $s$-coding has already been determined. Figure 1 shows an example of s-coding. In this figure, $T 0$ and $T 1$ are ordered forms of two database trees, and the trees below them show the $s$-codings of their nodes.
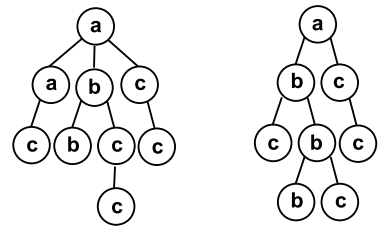

Input tree To


S-coding of To

S-coding of $\mathrm{T}_{1}$

Figure 1: An example of s-coding.

\section{is-coding}

First, we introduce the r-preorder traversal.

Definition 4: R-preorder traversal of a rooted ordered tree is a depth-first traversal which is defined recursively as follows:

1) visit the root first; and then

2) do an r-preorder traversal each of the subtrees of the root one-by-one from right to left.
In r-preorder traversal, the children are met from right to left which is the key difference compared to the preorder traversal.

Definition 5: Let $T$ be a database tree, $\prec$ be a left-toright ordering among children of nodes of $T$, and sid be an auxiliary integer initiated by 0 . For every node $v \in V(T)$, its $i$-coding with respect to $\prec$, denoted by $\mathcal{I} \mathcal{S}_{\prec}(T, v)$ is defined as follows:

1) if $v$ is the root of $T, \mathcal{I} \mathcal{S}_{\prec}(T, v)$ is defined equal to 0 .

2) $T_{\prec}$ is traversed in r-preorder and when a node $v$ is met, if $\operatorname{deg}(T, v)>1$, sid is increased by 1 , and for any child $u$ of $v, \mathcal{I S}_{\prec}(T, u)$ is defined equal to the new value of sid.

$s$-coding and is-coding only differ in the way of traversing ordered forms of database trees. Figure 2 shows the isencoding of the ordered forms of the database trees presented in Figure 1.

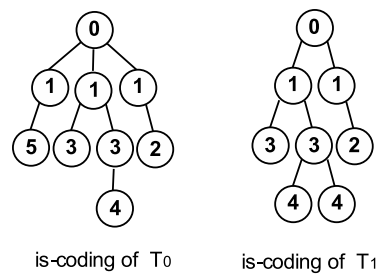

Figure 2: An example of is-coding.

cs-coding

Definition 6: Let $\prec$ be a left-to-right ordering among children of nodes of $T$. The cs-coding of a node $v$ in a database tree $T$ with respect to $\prec$, denoted by $\mathcal{C S}_{\prec}(T, v)$, is defined as $\mathcal{S C}_{\prec}$ of its children.

When a node is met in preorder traversal of the tree, the $s$-codings of its children are assigned, therefore $c s$-coding of all nodes (with respect to an ordering $\prec$ ) can be determined in $O(|V(T)|)$. Figure 3 shows the cs-encoding of the ordered forms of the database trees presented in Figure 1.

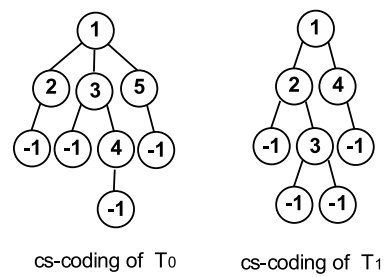

Figure 3: An example of cs-coding.

\section{The frequency counting method}

Each candidate $C_{k+1}$ can be generated by either rp_extension or rn_extension. In this section, we present new frequency counting methods for rp_extended candidates 
and rn_extended candidates, based on the tree encodings presented in Section III-B.

The following notions and notations are used in this section: we assume that $C_{k}$ and $C_{k}^{\prime}$ are two trees belonging to the same equivalence class, and $C_{k} \odot C_{k}^{\prime}$ is possible and it generates the tree $C_{k+1} \cdot C_{k+1}$ is generated by adding the node $N$ to $C_{k}$. We also assume that the occurrence $O_{k}$ of $C_{k}$, the occurrence $O_{N}$ of $N$, and the occurrence $O_{k+1}$ of $C_{k+1}$ all occur in the database tree $T$. $C N$ refers to the rightmost node of $C_{k}$ and $C P$ refers to the rightmost path of $C_{k}$ excluding its rightmost node, i.e. $C P \cup C N$ forms the rightmost path of $C_{k} . O_{C N}$ is the node in $O_{k}$ corresponding to the node $C N$ of $C_{k}$, and $O_{C P}$ is the path in $O_{k}$ corresponding to $C P$. We note that since $C_{k}$ is an unordered occurrence of $C_{k}$, it is possible that $O_{C N}$ and $O_{C P}$ are not the rightmost node and the rightmost path of $O_{k}$, respectively.

\section{Frequency counting for rp_extended candidates}

$C_{k+1}$ is generated by adding node $N$ to $C_{k}$ via rp_extension and we want to see whether or not adding $O_{N}$ to a node in $O_{C P}$ generates an occurrence $O_{k+1}$ of $C_{k+1}$. $T$ can be divided into the partitions depicted in Figure 4. $B 1$ is the path between the root of $T$ and the root of $O_{k} . C$ contains the children of the nodes of $B 1$ and the children of the nodes of $O_{C P} . B 2$ is the path between $O_{C N}$ and $z$, where $z$ is the last node met before $O_{C N}$ in preorder traversal of $T$. To generate an occurrence $O_{k+1}$ of $C_{k+1}$, $O_{N}$ must belong to the dotted region depicted in Figure 4. For this purpose, $O_{N}$ must satisfy Properties 1, 2 and 3.

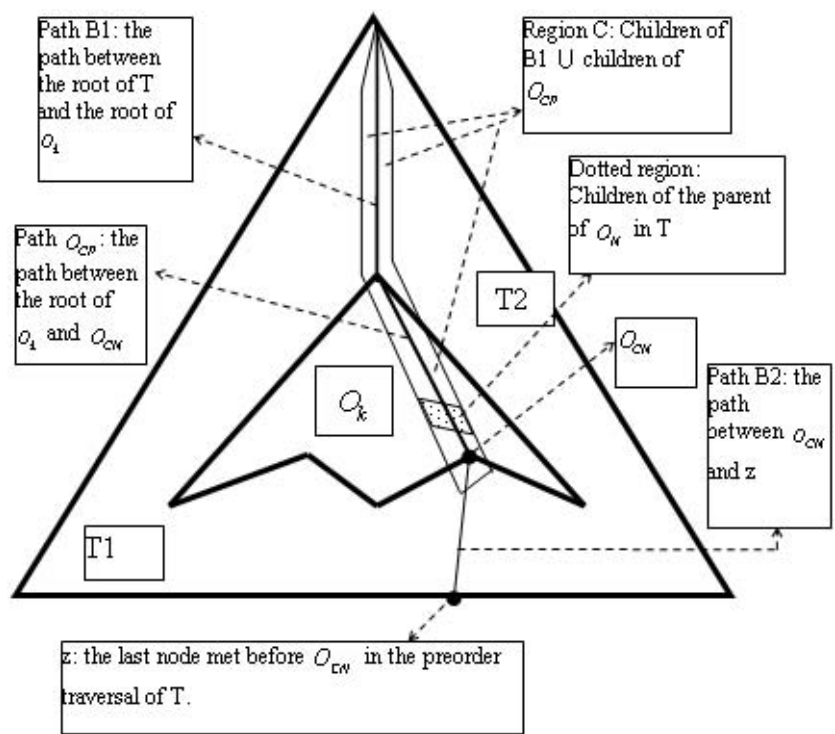

Figure 4: A partitioning of a database tree.

Property 1: $\mathcal{S C}_{\prec}\left(O_{N}\right) \leq \mathcal{S C}_{\prec}\left(O_{C N}\right)$.
Property 2: $\mathcal{I S}_{\prec}\left(O_{N}\right) \leq \mathcal{I S}_{\prec}\left(O_{C N}\right)$.

where $\prec$ is any arbitrary ordering on the nodes of the database tree.

$O_{N}$ can be anywhere in $T$. It can be shown that if Property 1 is applied on $O_{N}$, it can not be selected from the grey parts of Figure 5(a). We also can show that Property 2 expels $O_{N}$ from the grey parts of Figure 5(b). The intersection of non-grey parts of Figures 5(a) and 5(b) is the region $C$, i.e. applying Properties 1 and 2 to $O_{N}$, restricts it to $C$. Property 3 restricts $O_{N}$ to the dotted region, which is the desired region.

Property 3: $\operatorname{dep}\left(T, O_{N}\right)-\operatorname{dep}\left(T, O_{C N}\right)=\operatorname{dep}\left(C_{k}, N\right)-$ $\operatorname{dep}\left(C_{k}, C N\right)$.

If the nodes in a database tree are allowed to have duplicate labels, but the labels for the children of every node are assumed to be unique, Properties 1,2 and 3 are sufficient to count frequencies of rp_extended candidates. Assume that $C_{k+1}$ is generated by adding node $N$ to $C_{k}$ via rp_extension. To see whether adding $O_{N}$ to the rightmost node of $O_{C_{k}}$ generates $O_{k+1}$, it is sufficient to check if:

1) $O_{N}$ and $O_{k}$ occur in the same database tree,

2) $\mathcal{S C}_{\prec}\left(O_{N}\right) \leq \mathcal{S C}_{\prec}\left(O_{C N}\right)$,

3) $\mathcal{I S}_{\prec}\left(O_{N}\right) \leq \mathcal{I} \mathcal{S}_{\prec}\left(O_{C N}\right)$, and

4) $\operatorname{dep}\left(T, O_{N}\right)-\operatorname{dep}\left(T, O_{C N}\right)=\operatorname{dep}\left(C_{k}, N\right)-$ $\operatorname{dep}\left(C_{k}, C N\right)$.

( $\prec$ is any arbitrary ordering on the nodes of database trees.)

If these conditions are satisfied, $O_{k+1}$ is generated by attaching $O_{N}$ to $O_{k}$. s-coding, is-coding and cs-coding of $O_{N}$ are stored for $O_{k+1}$ to be used in future extensions of $O_{k+1}$.

As an example, consider tree "b b - $1 \mathrm{c}$ " which is generated by $b \quad b \odot b c$. As depicted in Figure 6 , "b b" has one occurrence in $T 0$ and two occurrences in $T 1$. Node "c" occurs for 5 times in $T 0$ and for 4 times in $T 1$. Figure 6 shows how the occurrences of "b b -1 c" can be determined by means of occurrences of " $b$ b" and "c". Left side trees highlight the occurrences of " $b$ " and right side trees show different occurrences of "c". Texts on the side of the hachured occurrences of "c" present the unsatisfied properties. Other occurrences of "c", depicted by double circles, are occurrences that satisfy all the conditions; therefore they can join with an occurrence of " $b$ b" and generate an occurrence of "b b - 1 c".

\section{Frequency counting for rn_extended candidates}

The following theorem can help us to count frequencies of rn_extended candidates either when database trees have unique labels on the children of every node or when they are general unordered trees.

Theorem 1: Assume that $C_{k}$ is generated by adding node $N$ to $C_{k}$ via rn_extension. To see whether adding $O_{N}$ to the rightmost node of $O_{k}$ generates $O_{k+1}$, it is sufficient to check if:

1) $O_{N}$ and $O_{k}$ occur in the same database tree, and 


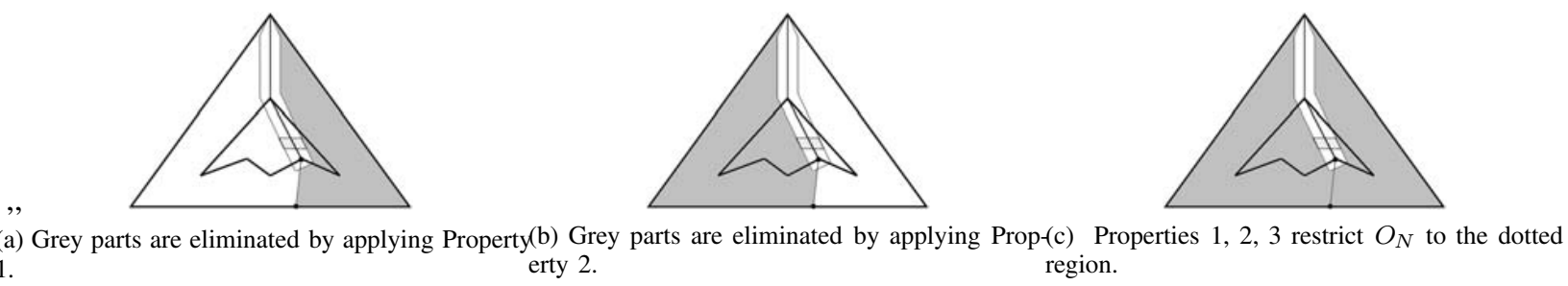

Figure 5: Restrictions applied by of Properties 1, 2 and 3 on the nodes of the database tree $T$.

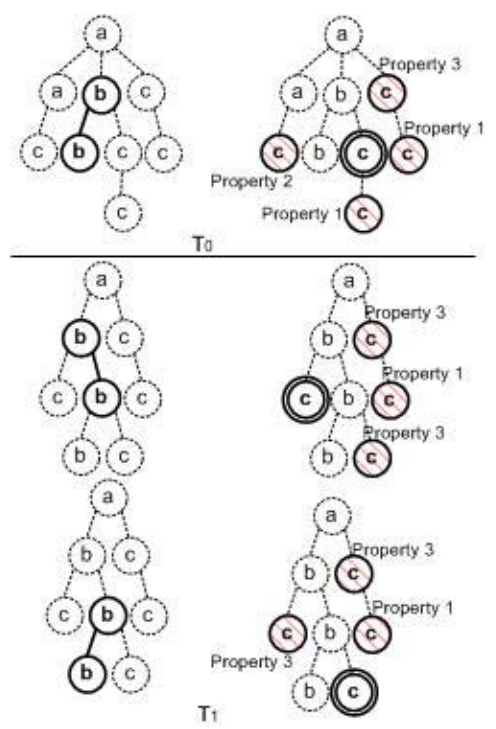

Figure 6: An example of frequency counting for rp_extended candidates.

\section{2) $\mathcal{C S}_{\prec}\left(O_{C N}\right)=\mathcal{S C} \prec\left(O_{N}\right)$.}

where $\prec$ is any arbitrary ordering among the nodes of the database tree.

If the conditions of Theorem 1 are satisfied, $O_{k+1}$ is generated and the s-coding, is-coding and cs-coding of $O_{N}$ are stored for $O_{k+1}$ to be used in future extensions of $O_{k+1}$.

Figure 7 shows an example of frequency counting for rn_extended candidates. Rn_extension of "a b" by means of "c" generates the tree "a b c". As depicted in Figure 7, "a b" has one occurrence in $T 0$ and one occurrence in $T 1$. For each occurrence, only one occurrence of "c" satisfies both conditions. Therefore, "a b c" have two occurrences in the database trees.

\section{Generalizing the Frequency Counting Method}

In this section, we assume that the database trees are general trees and their nodes can have children with the same label. For frequency counting of rn extended candidates, this generalization does not make any problem; however, for rp_extended candidates we must revise the frequency counting algorithm.

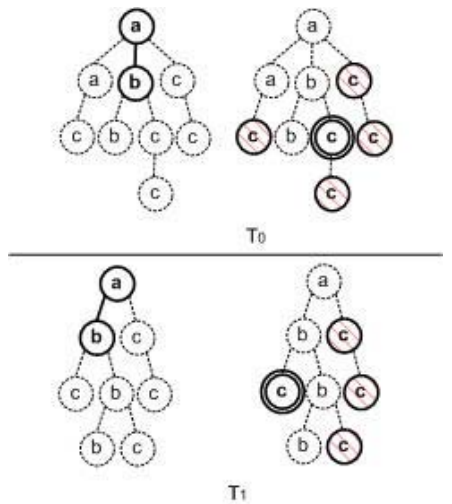

Figure 7: An example of frequency counting for rn_extended candidates.

For generalized rp_extended frequency counting, it is possible that $O_{N}$ belongs to the dotted region of Figure 4 and it has already been appended to $O_{k}$. In this case, the above mentioned frequency counting method is not enough restricting for selecting correct occurrences of $O_{N}$.

Definition 7: Tree candidate $C^{\prime}$ is self-extended if it is generated by rp_extension of a candidate $C$ " with itself. In the consequence of this extension, which is called selfextension, the rightmost node and the second rightmost node $^{1}$ of the resultant candidate find the same label. This same label is called self-extension label.

Theorem 2: For self-extended candidates, to guarantee that $O_{N}$ has not already been appended to the dotted region of $C_{k}$, more than the 4 conditions already mentioned, the following condition should also be held:

$$
\mathcal{P}\left(T_{\prec}, O_{C N}\right)<\mathcal{P}\left(T_{\prec}, O_{N}\right)
$$

where for a node $v \in V\left(T_{\prec}\right), \mathcal{P}\left(T_{\prec}, v\right)$ is the preorder number of $v$ in $T_{\prec}$.

In this case, in order to correctly count frequencies of candidates extended from $C_{k}$, both the encodings of $O_{N}$ and the encodings of $O_{C N}$ are stored for $C_{k}$, if they have not already been stored.

\footnotetext{
${ }^{1}$ The second rightmost node is the rightmost node of the tree generated from removing the rightmost node.
} 
As an example, consider Figure 8 in which the database tree is "c $\mathrm{b}$ d $-1 \mathrm{a}-1 \mathrm{~b}-1 \mathrm{a}-1 \mathrm{~b}-1$ " and we want to compute the frequency of "c $b \mathrm{~d}-1 \mathrm{~b}-1 \mathrm{~b}-1$ ". "c $\mathrm{b} d-1 \mathrm{~b}-1 \mathrm{~b}-1$ " is a self-extended candidate which is generated by appending node "b" to "c b d -1 b - 1 ". "c b d -1 b -1" has two occurrences in the database tree. For the first occurrence, the rightmost occurrence of " $b$ " satisfies the condition of Theorem 2 as well as all of the 4 conditions already mentioned for rp_extended candidates. For the second occurrence, none of the occurrences of "b" satisfy the condition of Theorem 2 . Therefore, "c b d -1 b $-1 \mathrm{~b}-1$ " has one occurrence in the database tree.
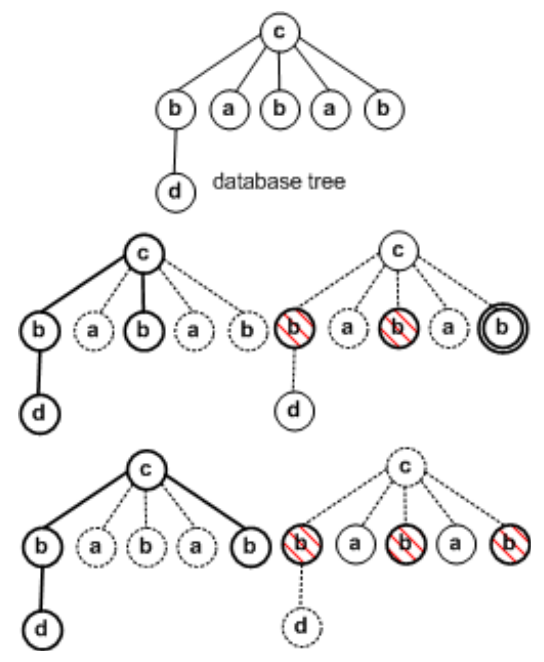

Figure 8: An example of generalized frequency counting for self-extended candidates.

Theorem 2 solves the problem for self-extended candidates, however, the problem still remains for other rp_extended candidates.

Assume that candidate $C_{k+1}=C_{k} \odot C_{k}^{\prime}$ is generated via rp_extended. In every occurrence $O_{k}^{\prime}$ of $C_{k}^{\prime}$, we refer to the image of $N$ by $O_{N}: O_{k}^{\prime}$. Without going into details and proofs (due to lack of space), we present Theorem 3 for frequency counting of $\mathrm{rp}$ _extended candidates whih are not self-extended.

Theorem 3: Frequency of a rp_extended candidate $C_{k+1}=C_{k} \odot C_{k}^{\prime}\left(C_{k+1}\right.$ is not self-extended $)$ in a database tree $T$ can be correctly computed as the following: if:

for every two occurrences $O_{k}$ of $C_{k}$ and $O_{k}^{\prime}$ of $C_{k}^{\prime}$ in $T$,

1) $O_{N}: O_{k}^{\prime}$ is not the rightmost node of any already generated occurrence of $C_{k+1},{ }^{2}$

2) $\mathcal{C S}_{\prec}\left(O_{N}: O_{k}^{\prime}\right) \neq \mathcal{S C}_{\prec}\left(O_{C N}\right)$,

3) $\mathcal{S C}_{\prec}\left(O_{N}: O_{k}^{\prime}\right) \leq \mathcal{S C} \prec\left(O_{C N}\right)$,

\footnotetext{
${ }^{2}$ Since the occurrences of a candidate tree can be generated in an ordered manner, this condition can be tested very efficiently by only 1 comparison. Due to lack of space we omit details.
}

4) $\mathcal{I S}_{\prec}\left(O_{N}: O_{k}^{\prime}\right) \leq \mathcal{I} \mathcal{S}_{\prec}\left(O_{C N}\right)$, and

5) $\operatorname{dep}\left(T, O_{N}: O_{k}^{\prime}\right)-\operatorname{dep}\left(T, O_{C N}\right)=\operatorname{dep}\left(C_{k}, N\right)-$ $\operatorname{dep}\left(C_{k}, C N\right)$.

an occurrence $O_{k+1}$ of $C_{k+1}$ is generated ( $\prec$ is an arbitrary ordering on the nodes of database trees).

For example, in Figure 9 "c $\mathrm{b} d \mathrm{~d}-1-1 \mathrm{~b}-1 \mathrm{~b}-1$ " and "c b d -1 -1 b -1 a -1" join together and generate "c b $\mathrm{d}-1-1 \mathrm{~b}-1 \mathrm{~b}-1$ a -1 ". " c $\mathrm{b}$ d $-1-1$ b -1 a -1 " has 4 occurrences in the database tree "c $\mathrm{b} d \mathrm{~d}-1-1 \mathrm{a}-1 \mathrm{~b}-1$ $\mathrm{a}-1 \mathrm{~b}-1$ ". In the first and third occurrences, occurrence "a" satisfies all the above mentioned conditions. In the second occurrence, occurrence "a" is the same as in the first occurrence, and in the forth occurrence, occurrence "a" is the same as in the third occurrence. Therefore, occurrence "a" in the second and forth occurrences can not participate in the join operation.

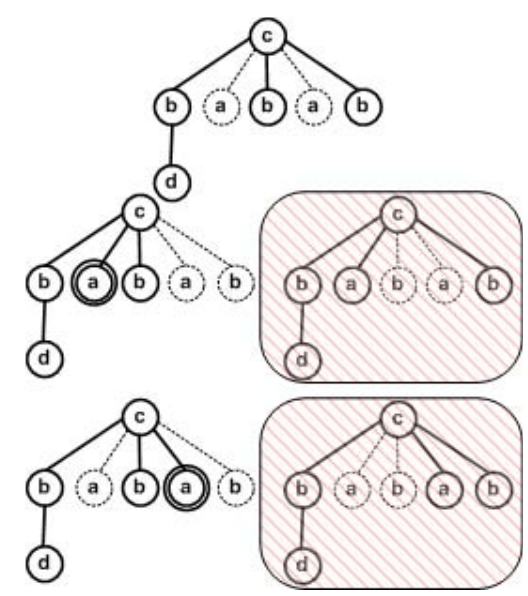

Figure 9: An example of frequency counting for non self_extended candidates in which nodes might have children with the same labels.

\section{E. The UITree Algorithm}

Figure 10 shows the high level pseudo code of the UITree algorithm. First, the set of frequent labels (frequent patterns of size 1) are generated. Then, larger patterns are generated via recursive calls of the REFINEMENT method.

In the REFINEMENT method, a pattern is extended if it is in the canonical form. Here, we use the canonical form presented in [29]. If a pattern $C$ is in the canonical form, it is rn_extended using frequent labels and frequency of every resultant candidate tree is computed according to Theorem 1; and $C$ is rp_extended using patterns which are equivalent to it and frequency of every resultant candidate tree is computed according to Theorems 2 and 3.

To guarantee the completeness of the candidate generation step, $C$ is rp_extended by every pattern $C^{\prime}$ which is in the equivalence class of $C$ (either $C^{\prime}$ is in the canonical form or is not). In this way of candidate generation, only 
known frequent patterns are used to rp_extend $C$ ( $C$ itself is frequent). In this method, candidates which are frequent but are not in the canonical form are used for rp_extension of other candidates.

Another possible way for candidate generation is generating only candidates which are in the canonical form and rp_extending them by all frequent labels. In this way, it is possible that a candidate which is already known to be infrequent (since it has an infrequent subtree), is generated.

As discussed in [29], there is a trade-off between the number of redundant candidates (which are not in the canonical form) generated in the first method, and the number of already known infrequent candidates generated in the second method. In [29] these two different methods were experimentally evaluated, and it was found that the first method of candidate generation is more efficient than the second method. We note that at the end, both methods generate the same set of frequent patterns.
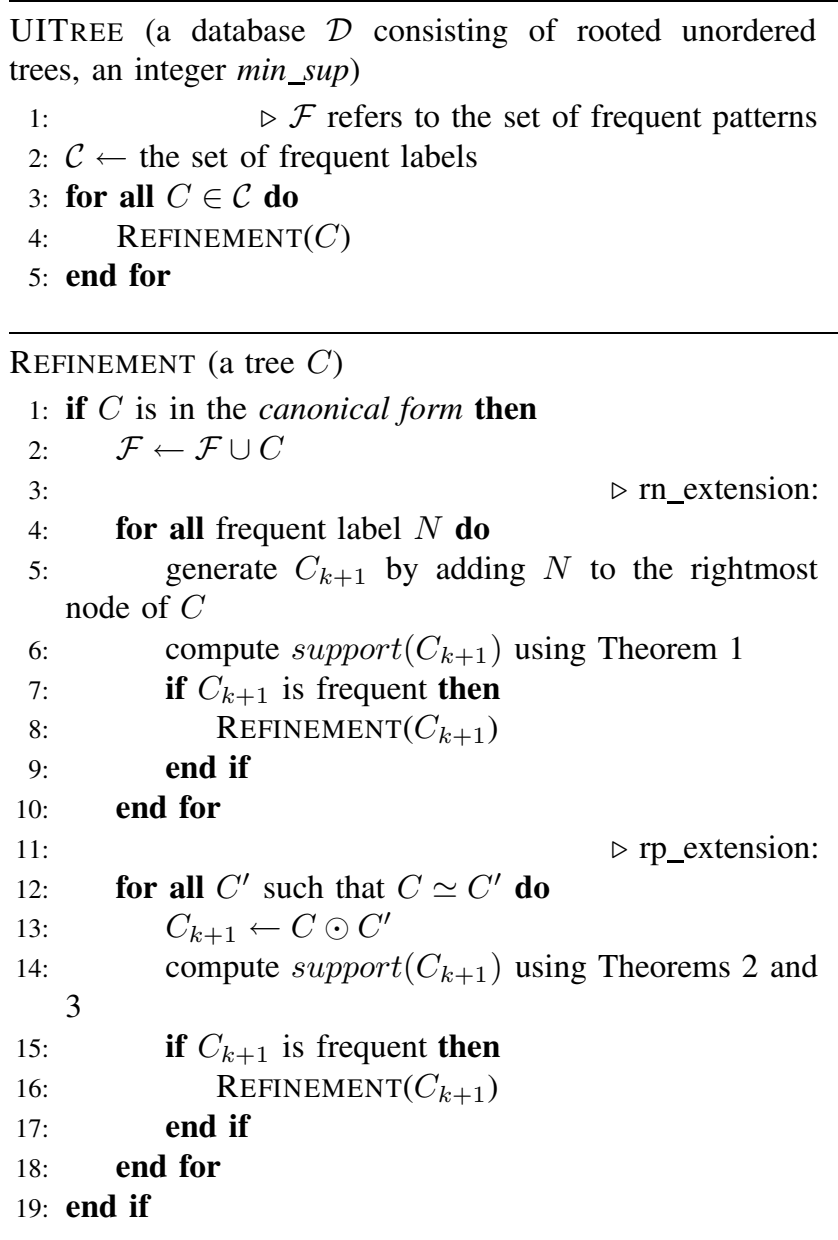

Figure 10: High level pseudo code of UITree.

\section{EXPERIMENTAL RESULTS}

We perform extensive experiments to evaluate the efficiency of the proposed algorithm using data from real applications as well as synthetic datasets. We do our experiments on a $2.93 \mathrm{GHz}$ Intel Pentium IV PC with a $8 \mathrm{~GB}$ main memory, running UNIX operating system. All the algorithms are implemented in $\mathrm{C}++$ using standard template libraries.

The well-known algorithm in the literature for mining unordered trees is HybridTreeMiner [6]. [7] and [3] have been reported to be more efficient, but they find frequent closed and maximal patterns. Here, we select HybridTreeMiner for our comparisons.

The CSLOGS dataset [30] is a real dataset which contains the web access trees of the CS department of the Rensselaer Polytechnic Institute during one month. This dataset has 59,691 transactions, 716,263 nodes and 13,209 unique vertex labels. Each distinct label corresponds to the URLs of a web page.

In [31], the log file of every week is separated into a different dataset and 3 different datasets are generated: CSLOG1 for the first week, CSLOG2 for the second week and CSLOG3 for the third week. Furthermore, they generated a new dataset called CSLOG12 by combining CSLOG1 and CSLOG2. CSLOG1 contains 8,074 trees, CSLOG2 contains 7,404 trees, CSLOG3 contains 7,628 trees, and CSLOG12 contains 13,934 trees. We use these real datasets to evaluate the efficiency of the proposed algorithm.

Figure 11 compares UITree against HybridTreeMiner over CSLOG1. As depicted in the figure, UITree significantly outperforms HybridTreeMiner, for example in min_sup = 13, UITree outpeforms HybridTreeMiner by a factor of 300 (the left side diagram). Two algorithms use almost equal amounts of memory (the right side diagram).

Figures 12, 13 and 14 compare UITree against HybridTreeMiner over CSLOG2, CSLOG12 and CSLOG3, respectively. Over CSLOG2, in min_sup $=10$, НybridTreeMiner can not generate the output after several hours. In min_sup $=12$, UITree works more than 250 times faster than HybridTreeMiner. Over CSLOG12, for the values of min_sup smaller than 21, HybridTreeMiner can not generate the output after a reasonable time (several hours). In min_sup $=21$, UITree works more than 200 times faster than HybridTreeMiner. Over CSLOG3, for the values of min_sup smaller than 15, HybridTreeMiner can not generate the output after a reasonable time. In min_sup $=15$, UITree works more than 150 times faster than HybridTreeMiner.

We also evaluate the efficiency of the proposed algorithm using synthetic datasets which are generated by the method described in [30]. The synthetic data generation program mimics the web site browsing behavior of the user. First a master web site browsing tree is built and then the subtrees of the master tree are generated. The synthetic 

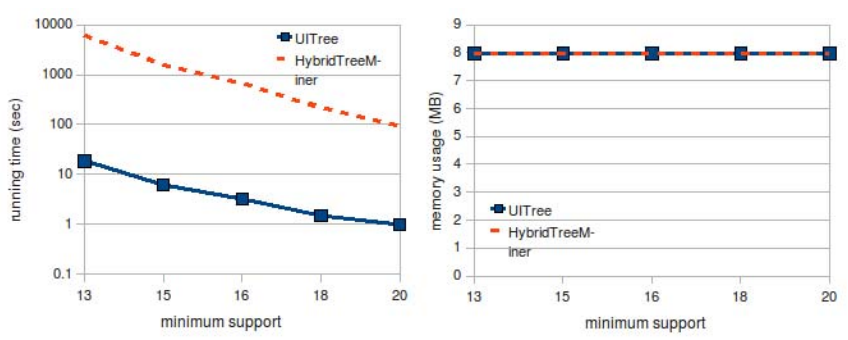

Figure 11: Comparison over CSLOG1.
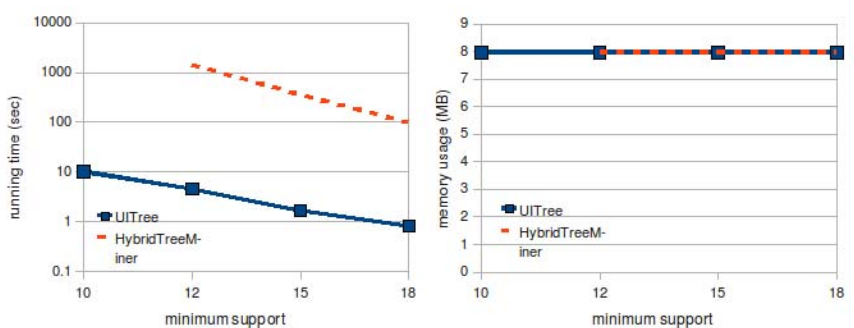

Figure 12: Comparison over CSLOG2.

tree generation program is adjusted by 5 parameters: 1) the number of labels $(N), 2)$ the number of nodes in the master tree $(M), 3)$ the maximum fan-out of a node in the master tree $(F), 4)$ the maximum depth of the master tree $(D)$, and $5)$ the total number of trees in the dataset $(T)$.

The first synthetic dataset is D10 and uses the following default values for the parameters: $N=100, M=10,000$, $D=10, F=10, T=100,000$. Figure 15 compares two algorithms on D10. As depicted in the figure, UITree always outperforms HybridTreeMiner.

F3 uses the default value expect for $F=3$. As depicted in Figure 16, over this dataset, UITree works faster than HybridTreeMiner. M100K uses the default values expect for $M=100,000$. Figure 17 reports the efficiency of UITree compared to HybridTreeMiner over this dataset.

In N10K, $N$ is set to 10,000. In Figure 18, we compare two algorithms over this dataset, and as depicted in the diagram, UITree significantly outperforms HybridTreeMiner. For example, in min_sup $=4$, UITree works more than 35
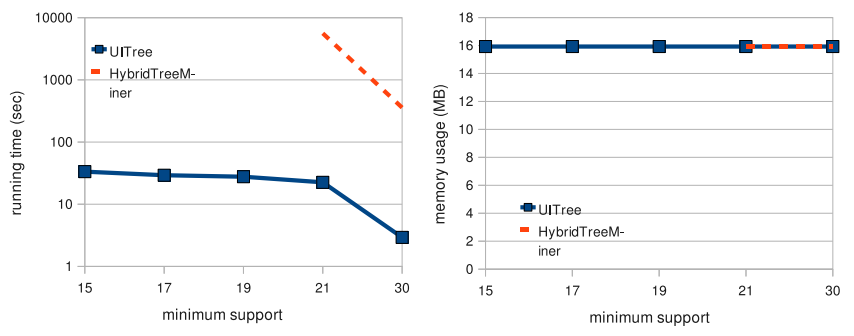

Figure 13: Comparison over CSLOG12.
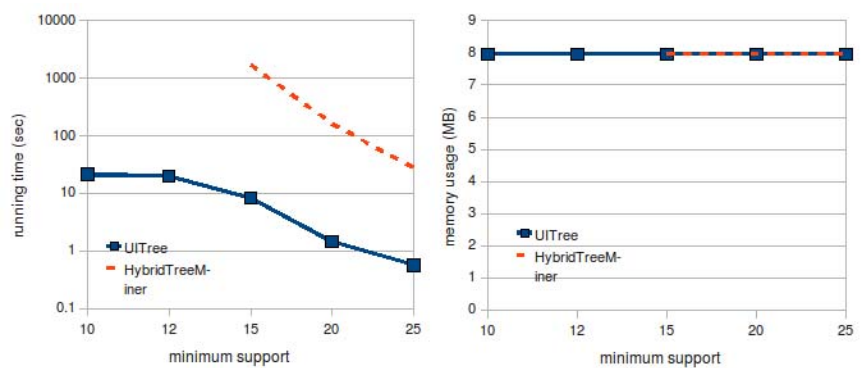

Figure 14: Comparison over CSLOG3.
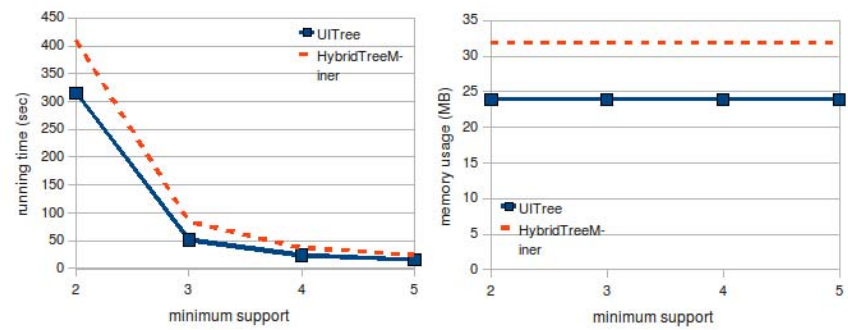

Figure 15: Comparison over D10.

times faster than HybridTreeMiner.

\section{RELATED WORKS}

Recently, many algorithms have been proposed to find frequent patterns from a collection of database trees. Wang et al. proposed algorithms for discovering similar structure and structural association rules among a collection of tree structured data [22], [23] and [24].

Zaki introduced TreeMiner [28] to mine embedded ordered frequent tree patterns. For frequency counting, he used an efficient data structure, called scope-list, and proposed rightmost path extension to generate non-redundant candidates. Later, by proposing the SLEUTH algorithm, he extended the work to find embedded patterns from rooted unordered [29]. Asai et al. [1] independently proposed the rightmost candidate generation and developed FREQT for mining frequent induced ordered tree patterns. Chi et al. [5] proposed FreeTreeMiner for mining induced unordered free
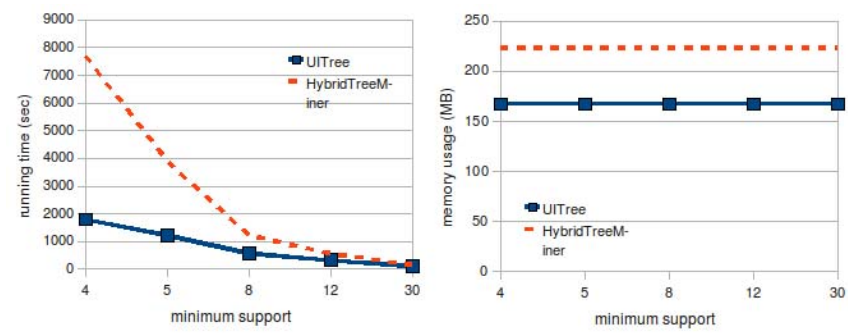

Figure 16: Comparison over F3. 

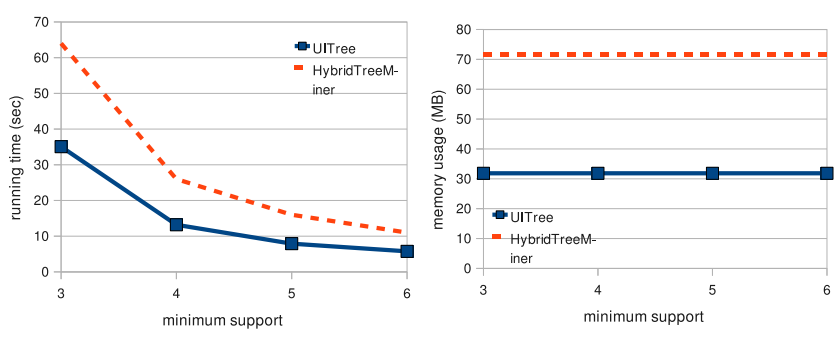

Figure 17: Comparison over M100K.
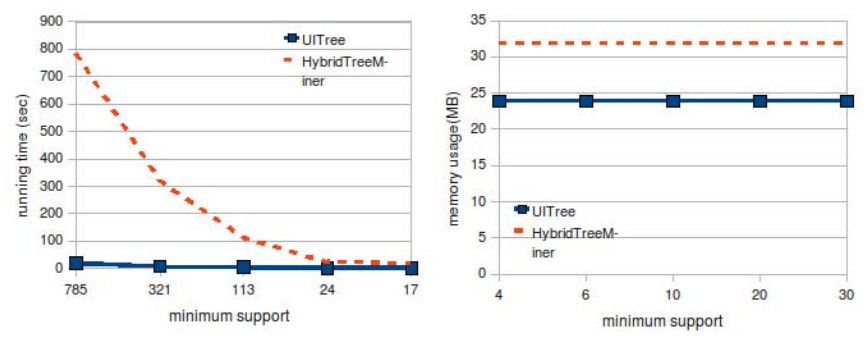

Figure 18: Comparison over N10K.

trees. Other algorithms for mining induced unordered tree patterns are PathJoin [25], uFreqt [14], uNot [2] and HybridTreeMiner [6]. TreeFinder [20] uses an Inductive Logic Programming approach to mine unordered embedded trees but it is not a complete method and can miss some frequent subtrees. SingleTreeMining [15] is an algorithm for mining rooted unordered trees with application to phylogenetic tree pattern mining.

Chi et al. proposed CMTreeMiner [7] for mining both closed and maximal frequent subtrees in a database of rooted unordered trees. This algorithm traverse an enumeration tree that systematically enumerates all subtrees. Xiao et al. [26] proposed TreeGrow for mining unordered maximal embedded tree patterns. However, TreeGrow assumes that the labels for the children of every node are unique. The candidate generation method is localized so as to avoid unnecessary computational overhead. [13] and [16] developed other algorithms for finding maximal embedded tree patterns.

XSpanner [21] is a pattern growth-based method and can mine embedded ordered subtrees. Tatikonda et al. [18] proposed a generic approach that can be used to mine embedded or induced subtrees that can be labeled, unlabeled, ordered, unordered, or edge-labeled. They developed TRIPS and TIDES algorithms using two sequential encodings of trees to systematically generate and evaluate the candidate patterns. Tan et al. [17] presented a unique embedding list representation of the tree structure, which enables efficient implementation of their Tree Model Guided (TMG) candidate generation. TMG can enumerate all the valid candidates that conform to the structural aspects of the input data.
UNI3 [8] generates unordered candidate while it performs ordered frequency counting. In [19], the authors developed efficient pattern mining algorithms specialized for multi-core hardwares.

For finding unordered frequent tree patterns, most of the proposed algorithms use a canonical form and extend only candidates that are in the canonical form. In [11] and [12], Luccio et al. defined sorted pre-order string canonical form can be obtained in linear time (assuming a finite alphabet for vertex labels). Later, Asai et al. [2], Nijssen et al. [14], and Chi et al. [5] independently defined similar canonical representations.

Efficient algorithms for mining frequent graph patterns which are the general form of frequent tree patterns can be found in [9], [10] and [27].

\section{CONClusion AND FUture WORKS}

In this paper, we presented novel tree encodings, and accordingly, developed a new method for finding frequent patterns from rooted unordered trees with unique labels on children of every node. Then, we generalized the method to find frequent patterns from rooted unordered trees without any restriction. We empirically evaluated the efficiency of the proposed algorithm, UITree, and showed that it provides significant improvements against well-known algorithms in the literature, over both real and synthetic datasets.

One future work is extending the proposed algorithm to find condensed representations like frequent closed patterns. A useful technique which is widely used (in different forms) to trim the state space in the closed pattern mining problem, is the early termination or early prunning technique [7] and [27]. This technique can be performed more efficiently using the encodings and the frequency counting method presented in this paper. The other future work, can be developing similar techniques for fining frequent patterns from more complex graphs such as plannar graphs, bounded-treewidth graphs, or general graphs.

\section{ACKNOWLEDGMENT}

We would thank to Dr Yun Chi for providing the HybridTreeMiner source code. We are also thankful to Professor Mohammed Javeed Zaki for providing the CSLOGS datasets and the TreeGenerator program. This work was partially supported by the ERC Starting Grant 240186 'MiGraNT'.

\section{REFERENCES}

[1] T. Asai, K. Abe, S. Kawasoe, H. Arimura, H. Satamoto and S. Arikawa, Efficient Substructure Discovery from Large SemiStructured Data, Proc. Second SIAM Intl Conf. Data Mining, pp. 158-174, Apr. 2002.

[2] T. Asai, H. Arimura, T. Uno and S. Nakano, Discovering Frequent Substructures in Large Unordered Trees, Proc. Sixth Intl Conf. Discovery Science, pp. 47-61, Oct. 2003. 
[3] J. L. Balczar, A. Bifet, A. Lozano, Mining Frequent Closed Rooted Trees, Machine Learning 78(1-2), 1-33 (2010).

[4] Mostafa H. Chehreghani, Morteza H. Chehreghani, C. Lucas, M. Rahgozar, OInduced: An Efficient Algorithm for Mining Induced Patterns From Rooted Ordered Trees, IEEE Transactions on Systems, Man and Cybernetics (A), 41(5), pp. 1013-1025, 2011.

[5] Y. Chi, Y. Yang and R.R. Muntz, Indexing and Mining Free Trees, Proc. Third IEEE Intl Conf. Data Mining (ICDM), pp. 509-512, 2003.

[6] Y. Chi, Y. Yang, and R.R. Muntz, HybridTreeMiner: An Efficient Algorihtm for Mining Frequent Rooted Trees and Free Trees Using Canonical Forms, Proc. 16th Intl Conf. Scientific and Statistical Database Management (SSDBM), pp. 11-20, 2004.

[7] Y. Chi, Y. Yang, Y. Xia, R. R. Muntz, CMTreeMiner: Mining Both Closed and Maximal Frequent Subtrees, PAKDD, pp. 6373, 2004.

[8] F. Hadzic and H. Tan and T. S. Dillon, UNI3-Efficient Algorithm for Mining Unordered Induced Subtrees Using TMG Candidate Generation, In Proceedings of IEEE International Symposium on Computational Intelligence and Data Mining (CIDM 07), 2007.

[9] A. Inokuchi, T. Washio and H. Motoda, An Apriori-based Algorithm for Mining Frequent Substructures from Graph Data, 4th European Conference on Principles of Knowledge Discovery and Data Mining, pp. 13-23, September 2000.

[10] M. Kuramochi and G. Karypis, Frequent Subgraph Discovery, Proceedings of the IEEE International Conference on Data Mining (ICDM01), pp. 313-320, November 2001.

[11] F. Luccio, A. M. Enriquez, P. O. Rieumont and L. Pagli, Exact Rooted Subtree Matching in Sublinear Time, Technical Report TR-01-14, Universita Di Pisa, 2001.

[12] F. Luccio, A. M. Enriquez, P. O. Rieumont and L. Pagli, Bottom-up Subtree Isomorphism for Unordered Labeled Trees, Technical Report TR-04-13, Universita Di Pisa, 2004.

[13] T. Miyahara, Y. Suzuki, T. Shoudai, T. Uchida, K Takahashi, H. Ueda, Discovery of Maximally Frequent Tag Tree Patterns with Contractible Variables from Semistructured Documents, PAKDD, pp. 133-144, 2004.

[14] S. Nijssen and J. N. Kok, Efficient Discovery of Frequent Unordered Trees, Proc. First Intl Workshop Mining Graphs, Trees, and Sequences, pp. 55-64, 2003.

[15] D. Shasha, J. Wang and S. Zhang, Unordered Tree Mining with Applications to Phylogeny, Proc. Intl Conf. Data Eng. (ICDE), pp. 708-719, 2004.

[16] Y. Suzuki, T. Miyahara, T. Shoudai, T. Uchida, Y. Nakamura, Discovery of Maximally Frequent Tag Tree Patterns with Height-Constrained Variables from Semistructured Web Documents, WIRI, pp. 104-112, 2005.
[17] H. Tan, F. Hadzic, T. S. Dillon, E. Chang, L. Feng, Tree Model Guided Candidate Generation for Mining Frequent Subtrees from XML Documents, ACM Transaction on Knowledge Discovery from Data (TKDD), 2(2), pp. 9-43, 2008.

[18] S. Tatikonda, S. Parthasarathy, T. M. Kur, TRIPS and TIDES: New Algorithms for Tree Mining, CIKM 06, pp. 455-464, 2006.

[19] S. Tatikonda, S. Parthasarathy, Mining Tree-Structured Data on Multicore Systems, PVLDB 2(1), 694-705, 2009.

[20] A. Termier, M. C. Rousset and M. Sebag, TreeFinder: a First Step towards XML Data Mining, Second IEEE International Conference on Data Mining (ICDM'02), p. 450, 2002.

[21] C. Wang, M. Hong, J. Pei, H. Zhou, W. Wang and B. Shi, Efficient Pattern-Growth Methods for Frequent Tree Pattern Mining, Proc. Pacific-Asia Conf. Knowledge Discovery and Data Mining, pp. 441-451, 2004.

[22] K. Wang and H. Liu, Schema Discovery for Semistructured Data, In Proc. of the 3rd. International Conference on Knowledge Discovery and Data Mining (KDD03), pp. 271-274, California, USA, August 1997.

[23] K. Wang and H. Liu, Discovering Typical Structures of Documents: A Road Map Approach, In Proc. of the ACM SIGIR International Conference on Research and Development in Information Retrieval (SIGIR), pp. 146-154, Melbourne, Australia, August 1998.

[24] K. Wang and H. Liu, Discovering Structural Association of Semistructured Data, IEEE Transactions on Knowledge and Data Engineering, 12(2), pp. 353-371, 2000.

[25] Y. Xiao, J. F. Yao, Z. Li and M. H. Dunham, Efficient Data Mining for Maximal Frequent Subtrees, Proc. Intl Conf. Data Mining (ICDM), pp. 379-386, 2003.

[26] Y. Xiao, J. F. Yao, G. Yang, Discovering Frequent Embedded Subtree Patterns from Large Databases of Unordered Labeled Trees, International Journal of Data Warehousing and Mining, 1(2), pp. 70-92, 2005.

[27] X. Yan and J. Han, CloseGraph: Mining Closed Frequent Graph Patterns, ACM SIGKDD Int. Conf. on Knowledge Discovery and Data Mining (KDD), pp. 286-295, August 2003.

[28] M. J. Zaki, Efficiently Mining Frequent Trees in a Forest, Proc of the Int. Conf. Knowledge Discovery and Data Mining (KDD), pp. 71-80, July 2002.

[29] M. J. Zaki, Efficiently Mining Frequent Embedded Unordered Trees, Fundam. Inform. 66(1-2), pp. 33-52, 2005.

[30] M. J. Zaki, Efficiently Mining Frequent Trees in a Forest: Algorithms and Applications, IEEE Transaction on Knowledge and Data Engineering, 17 (8), pp. 1021-1035, 2005.

[31] M. J. Zaki and C. C. Aggarwal, XRules: An Effective Structural Classifier for XML Data, Machine Learning, 62(1-2), pp. 137-170, 2006. 